Check for updates

Cite this: RSC Adv., 2017, 7, 22294

Received 4th April 2017

Accepted 18th April 2017

DOI: $10.1039 / \mathrm{c} 7 \mathrm{ra03846a}$

rsc.li/rsc-advances

\title{
Synthesis of carbon-supported Pd-Co bimetallic catalysts templated by Co nanoparticles using the galvanic replacement method for selective hydrogenation
}

\author{
Takeharu Yoshii, ${ }^{a}$ Kazuki Nakatsuka, ${ }^{a}$ Yasutaka Kuwahara, ${ }^{\text {ab }}$ Kohsuke Mori (D) abc \\ and Hiromi Yamashita (D) *ab
}

\begin{abstract}
Pd-Co bimetallic catalysts were prepared by the controlled synthesis of carbon-supported Co catalysts, ranging from single-sites to nanoparticles, via structural transformation of a deposited Co(salen) complex precursor by heat treatment at different temperatures, followed by galvanic replacement with Pd ions. The catalysts were structurally characterized using XRD, TEM, HAADF-STEM, XAFS and XPS. Highly dispersed Pd-rich NPs containing a low concentration of Co species were formed and the original size of the Co NPs was retained. The electronic state of the Pd species was dependent on the size of the bimetallic NPs, and a catalyst with a mean diameter of $10.8 \mathrm{~nm}$ was found to be in the most electronrich state. Not only the particle size, but also the electronic state of Pd play a crucial role in attaining high catalytic activity and selectivity in the selective hydrogenation of phenylacetylene. A Pd-Co catalyst based on a Co catalyst heat-treated at $600{ }^{\circ} \mathrm{C}$ showed $92 \%$ selectivity at $93 \%$ conversion. Furthermore, the $\mathrm{Pd}-\mathrm{Co}$ catalysts synthesized by the galvanic replacement method showed superior performance to a monometallic Pd catalyst and a Pd-Co alloy catalyst.
\end{abstract}

\section{Introduction}

Bimetallic nanoparticles (NPs) have attracted much attention over the last several decades due to their potential applications as catalysts. ${ }^{1-6}$ Ligand and ensemble effects in bimetallic catalysts have been reported to dramatically enhance their catalytic activity and selectivity. ${ }^{7-9}$ These effects depend on the composition, size, morphology and structure of the bimetallic species, ${ }^{10-12}$ and therefore control of these properties is important in the design of heterogeneous catalysts for specific reactions. Bimetallic nanostructures are generally categorized by alloy structure, heterostructure, and core-shell structure, and different nanostructures have been reported to show unique catalytic performance. ${ }^{\mathbf{1 3 , 1 4}}$ The preparation method is a crucial factor in determining the final structure of bimetallic NPs. ${ }^{15-17}$ Co-reduction of mixed metal ions is the most convenient method for preparing alloy NPs, whereas sequential deposition is typically used to prepare core-shell bimetallic NPs. A wide variety of additional strategies has been developed to synthesize bimetallic nanostructures. ${ }^{10,18}$

${ }^{a}$ Division of Materials and Manufacturing Science, Graduate School of Engineering, Osaka University, 2-1 Yamadaoka, Suita, Osaka 565-0871, Japan. E-mail: yamashita@mat.eng.osaka-u.ac.jp; Fax: +81-6-6105-5029; Tel: +81-6-6879-7457 ${ }^{b}$ Elements Strategy Initiative for Catalysts Batteries (ESICB), Kyoto University, Katsura, Kyoto 615-8520, Japan

'JST, PRESTO, 4-1-8 Honcho, Kawaguchi, Saitama 332-0012, Japan
The galvanic replacement (GR) method has been used over the last decade for the synthesis of bimetallic nanostructures. ${ }^{19,20}$ A pre-formed base metal, such as $\mathrm{Co}$, $\mathrm{Ni}$ or $\mathrm{Cu}$, and transition metal ions with a higher reduction potential, such as $\mathrm{Pd}^{2+}, \mathrm{Pt}^{2+}$ or $\mathrm{Au}^{3+}$, are used as precursors. The base metal is displaced by the transition metal ions due to the difference in ionization potential, and the second metal is redeposited onto the outer surface of the base metal. Various types of bimetallic nanostructures have been fabricated, including a core-shell structure, a hollow structure, and a porous structure, depending on the preparation conditions. A preparation technique based on GR has been developed for electro-catalysts, and various bimetallic catalysts show high electro-catalytic activities for oxygen reduction. ${ }^{21-27}$ The GR method has recently also been used to synthesize catalysts for other reactions, such as hydrogenation reaction, ${ }^{28-33}$ methane combustion $^{34}$ and preferential oxidation reaction. ${ }^{35}$

In this study, a series of carbon-supported Co species with different structures, ranging from single-sites to NPs, was used for the synthesis of Pd-Co by the GR method. Bimetallic nanoparticle catalysts prepared through the GR process usually exhibit core-shell ${ }^{21,26,32-34}$ or hollow/porous ${ }^{20,23,28,35}$ structures. In contrast, the Pd-Co NPs fabricated in this study are Pd-rich NPs with a low Co content that retain the size of the original Co NPs. Thus, the size of Pd-Co NPs can be controlled by the heat treatment temperature during preparation of the carbon- 
supported Co species. The electronic state of the Pd species was varied by changing the size of the Pd-Co nanoparticles, and the electron-rich Pd species was found to be most active and selective for the hydrogenation of phenylacetylene. In addition, the Pd-Co catalysts prepared by the GR method exhibited performance superior to that of monometallic Pd catalyst and Pd-Co catalysts prepared by the conventional impregnation method.

\section{Experimental}

\section{Materials}

Ethanol, methanol, 1,4-dioxane, acetone, styrene(monomer), ethylbenzene, biphenyl and sodium borohydride $\left[\mathrm{NaBH}_{4}\right]$ were purchased from Nakalai Tesque, Inc. $\left(\mathrm{CH}_{3} \mathrm{COO}\right)_{2} \mathrm{Co} \cdot 4 \mathrm{H}_{2} \mathrm{O}$ and $\mathrm{Pd}(\mathrm{II})$ nitrate solution were obtained from Wako Pure Chemical Ind. Co., Ltd. $N, N^{\prime}$-bis(salicylidene)-ethylenediamine $\left(\mathrm{H}_{2}(\mathrm{salen})\right)$ and ethynylbenzene phenylacetylene were purchased from Tokyo Chemical Industry Co., Ltd. Activated carbon (Shirasagi, M644) was obtained from Osaka Gas Chemicals Co., Ltd.

\section{Synthesis of Co(salen) complex}

The Co(salen) complex was prepared by a typical procedure. ${ }^{36}$ $\mathrm{H}_{2}$ (salen) (805 mg) was added into Ar-saturated ethanol $(10 \mathrm{~mL})$ and the solution was heated up to $90{ }^{\circ} \mathrm{C}$ in an oil bath. $\left(\mathrm{CH}_{3}{ }^{-}\right.$ $\mathrm{COO})_{2} \mathrm{Co} \cdot 4 \mathrm{H}_{2} \mathrm{O}(375 \mathrm{mg})$ as a Co precursor was added to the solution. After stirring for $24 \mathrm{~h}$, the solution was cooled, filtered and washed for removing the remaining $\mathrm{H}_{2}$ (salen) and $\mathrm{Co}^{2+}$ ions, followed by drying under vacuum for a day.

\section{Synthesis of Co/AC-T}

A series of carbon-supported Co catalysts were synthesized thorough the process we previously reported. ${ }^{37} \mathrm{Co}$ (salen) complex $(50 \mathrm{mg})$ was dissolved into acetone $(100 \mathrm{~mL})$ and the activated carbon (AC, $450 \mathrm{mg}$ ) was added to the solution. After stirring for $1 \mathrm{~h}$, the solvent was removed under vacuum with a rotary evaporator, and heat-treated at different temperatures for $2 \mathrm{~h}$ under a $100 \mathrm{~mL} \mathrm{~min}^{-1}$ nitrogen flow. The obtained powder was denoted as Co/AC- $T$ (Co: $1.8 \mathrm{wt} \%$ ), in which the $T$ indicates the heat treatment temperature.

\section{Synthesis of PdCo/AC-T-GR}

PdCo/AC-T-GR was prepared using GR method. Co/AC- $T$ (100 $\mathrm{mg}$ ) was dispersed in $20 \mathrm{~mL}$ of ethanol/ $\mathrm{H}_{2} \mathrm{O}$ and an aqueous solution of $\mathrm{NaBH}_{4}(4 \mathrm{~mL}, 75 \mathrm{mM})$ was added dropwise under vigorous stirring at $30{ }^{\circ} \mathrm{C}$ to reduce $\mathrm{Co}^{2+}$ to $\mathrm{Co}^{\circ}$. After $30 \mathrm{~min}$ stirring, the suspension was centrifuged and washed thoroughly with Ar-saturated ethanol/ $\mathrm{H}_{2} \mathrm{O}$. The product was dispersed in $20 \mathrm{~mL}$ of Ar-saturated ethanol/ $\mathrm{H}_{2} \mathrm{O}$, and $\mathrm{Pd}\left(\mathrm{NO}_{3}\right)_{2}$ aqueous solution $\left(140 \mu \mathrm{L}, \mathrm{Pd} 5 \mathrm{mg} \mathrm{mL}^{-1}\right)$ was added dropwise under continuous stirring at $30{ }^{\circ} \mathrm{C}$ for $30 \mathrm{~min}$. Then, the reaction of $\mathrm{Pd}^{2+}+\mathrm{Co}^{0} \rightarrow \mathrm{Pd}^{0}+\mathrm{Co}^{2+}$ occurred, and Pd NPs were formed by replacing Co species. Finally, the suspension was filtered and dried under vacuum for a day to obtain PdCo/AC- $T$ GR catalysts.

\section{Synthesis of Pd/AC}

The reference catalyst of $\mathrm{Pd} / \mathrm{AC}$ was synthesized by the following method. Activated carbon (100 mg) was dispersed in $20 \mathrm{~mL}$ of the ethanol/ $\mathrm{H}_{2} \mathrm{O}$ and $\mathrm{Pd}\left(\mathrm{NO}_{3}\right)_{2}$ aqueous solution $(140 \mu \mathrm{L}, \mathrm{Pd}$ $5 \mathrm{mg} \mathrm{mL}{ }^{-1}$ ) was added dropwise under continuous stirring. After string for $1 \mathrm{~h}$, the solvent was removed under vacuum with a rotary evaporator. The obtained powder was dried up for 1 day under vacuum and denoted as $\mathrm{Pd} / \mathrm{AC}$.

\section{Synthesis of PdCo/AC-600-imp}

The reference catalyst of PdCo/AC-600-imp was prepared using impregnation method. Co(salen) complex (10 mg) was dissolved into acetone $(20 \mathrm{~mL})$ and the activated carbon $(90 \mathrm{mg})$ was added to the solution. After stirring for $1 \mathrm{~h}$, the solvent was removed under vacuum with a rotary evaporator. The obtained powder was dispersed into ethanol $/ \mathrm{H}_{2} \mathrm{O}$, and $\mathrm{Pd}\left(\mathrm{NO}_{3}\right)_{2}$ aqueous solution $\left(140 \mu \mathrm{L}\right.$, Pd $\left.5 \mathrm{mg} \mathrm{mL}^{-1}\right)$ was added. After string for $1 \mathrm{~h}$, the solvent was removed under vacuum with the rotary evaporator. The obtained powder was heat-treated at $600{ }^{\circ} \mathrm{C}$ for $2 \mathrm{~h}$ under a $100 \mathrm{~mL} \min ^{-1}$ nitrogen flow, and will be referred as PdCo/AC-600-imp.

\section{Characterization}

Powder X-ray diffraction patterns (XRD) were measured using a Rigaku Ultima IV diffractometer with a semiconductor analyzer. The Pd K-edge X-ray absorption fine structure analysis (XAFS) spectra were recorded at the BL-01 at SPring-8, JASRI, Harima, Japan (Pro. No. 2016B1104 and 2016A1095). In XAFS measurements, a $\mathrm{Si}(111)$ double crystal was used to monochromatize the X-rays from the $2.5 \mathrm{GeV}$ electron storage ring. In the XAFS measurement, a sample was pretreated with hydrogen at $200{ }^{\circ} \mathrm{C}$ for $1 \mathrm{~h}$, loaded into the in situ cell with plastic windows and measured in fluorescence mode. The EXAFS data was examined using the EXAFS analysis program, Rigaku EXAFS. Transmission electron microscopy (TEM) images were obtained using a field emission-transmission electron microscope (FETEM; Hitachi Hf-2000) operated at $200 \mathrm{kV}$. Scanning transmission electron microscopy (STEM) images and elemental mapping were obtained using JEOL-ARM 200F equipped with a Kvex energy-dispersive X-ray detector (JED-2300T) operated at $200 \mathrm{kV}$. X-ray photoelectron spectroscopy (XPS) was performed with a Shimadzu XPS system, ESCA-3400, using the $\mathrm{Mg} \mathrm{K} \alpha$ line $(1253.6 \mathrm{eV})$. All samples were measured after hydrogen reduction at $200{ }^{\circ} \mathrm{C}$ for $1 \mathrm{~h}$, and the designated closed container was utilized for sample setting to prevent reoxidation. Binding energy was calibrated using the $\mathrm{C} 1 \mathrm{~s}$ photoelectronic peak at $284.5 \mathrm{eV}$.

\section{Catalytic test}

The catalytic performance was evaluated in the selective hydrogenation of phenylacetylene. Prior to the catalytic reaction, the catalyst were pretreated with hydrogen at $200{ }^{\circ} \mathrm{C}$ for $1 \mathrm{~h}$. Next, the suspension containing methanol $(5.0 \mathrm{~mL}), 1,4-$ dioxane $(5.0 \mathrm{~mL})$, phenylacetylene $(1.0 \mathrm{mmol})$, biphenyl $(0.5$ $\mathrm{mmol})$ as an internal standard and the catalyst $(10 \mathrm{mg})$ were 
added into a glass reactor. The reactor was placed in an oil bath at $30^{\circ} \mathrm{C}$, and the reaction was started by $10 \mathrm{~mL} \mathrm{~min}^{-1}$ hydrogen bubbling through the suspension under continuous stirring. The samples were examined by gas chromatography (Shimadzu, GC-2014 with TC-1 column).

\section{Results and discussion}

\section{Characterization}

The crystallinity of samples was investigated by XRD measurements. Fig. 1(A) shows XRD patterns for a series of Co/AC- $T$ precursors $(T=400,500,600$ and 800$)$. The broad peak at $40-$ $50^{\circ}$ for all samples originates from the amorphous structure of activated carbon. Co/AC-400 exhibited no significant peaks associated with Co species. In contrast, Co/AC-500, -600 and -800 showed clear peaks corresponding to metallic Co at $44.1^{\circ}$ or to $\mathrm{CoO}$ at $42.5^{\circ}$, and the intensity of the peaks increased with increasing heat treatment temperature, indicating the formation and aggregation of Co NPs above $500{ }^{\circ} \mathrm{C}$. XRD patterns for PdCo/AC-T-GR and PdCo/AC-600-imp are shown in Fig. 1(B). All samples were measured after pretreatment with hydrogen at $200{ }^{\circ} \mathrm{C}$ for $1 \mathrm{~h}$. PdCo/AC-T-GR $(T=400,500,600$ and 800) provided no peaks arising from Co species, unlike their Co/AC- $T$ counterparts, and no significant peaks derived from metallic Pd structure were observed (typically shown at $40.0^{\circ}$ and $46.6^{\circ}$ ). ${ }^{38}$ These results suggest that the crystallinity of Co NPs is reduced probably due to the atomic-scale replacement with Pd to form amorphous Pd-Co NPs. In contrast, PdCo/AC-600-imp showed a peak at around $41.5^{\circ}$, which is $1.5^{\circ}$ larger than that for the $\mathrm{Pd}$ face centered cubic (fcc) structure and $3.0^{\circ}$ lower than that for the Co fcc structure. This peak shift indicates the formation of a Pd-Co bimetallic alloy structure in PdCo/AC-600-imp. ${ }^{39,40}$

Fig. 2(A) shows transmission electron microscopy (TEM) images of Co/AC-T. No NPs are observed in Co/AC-400, suggesting the formation of single-site Co species on the support, as we previously demonstrated. ${ }^{37}$ In contrast, Co/AC-500 contained distinct NPs with a mean diameter of $5.9 \mathrm{~nm}$, and the $\mathrm{Co} /$

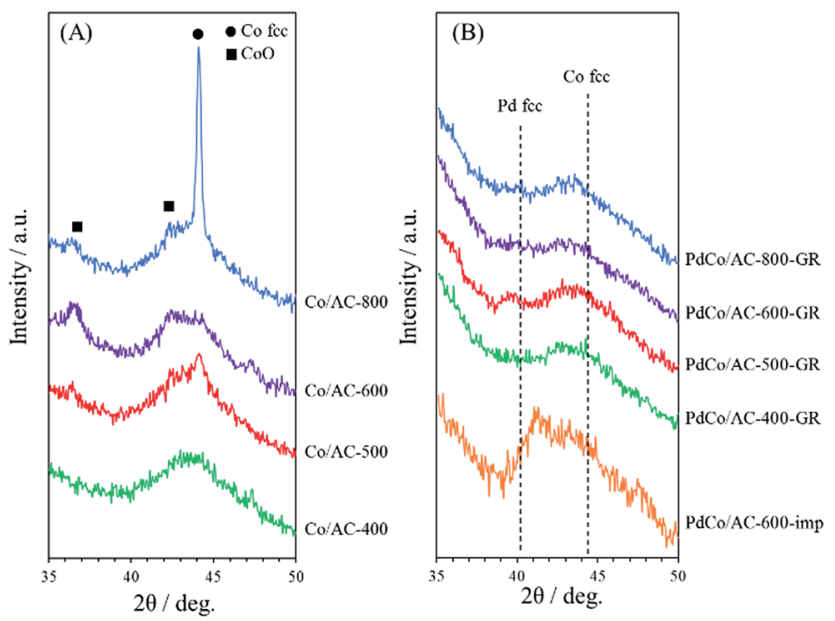

Fig. 1 XRD patterns of (A) Co/AC- $T(T=400,500,600,800)$ and (B) PdCo/AC-T-GR $(T=400,500,600,800)$ and PdCo/AC-600-imp.
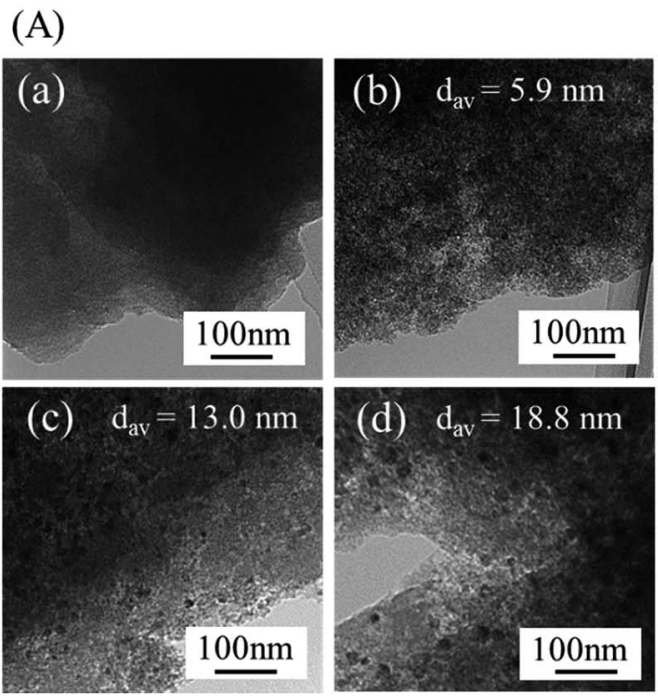

(B)
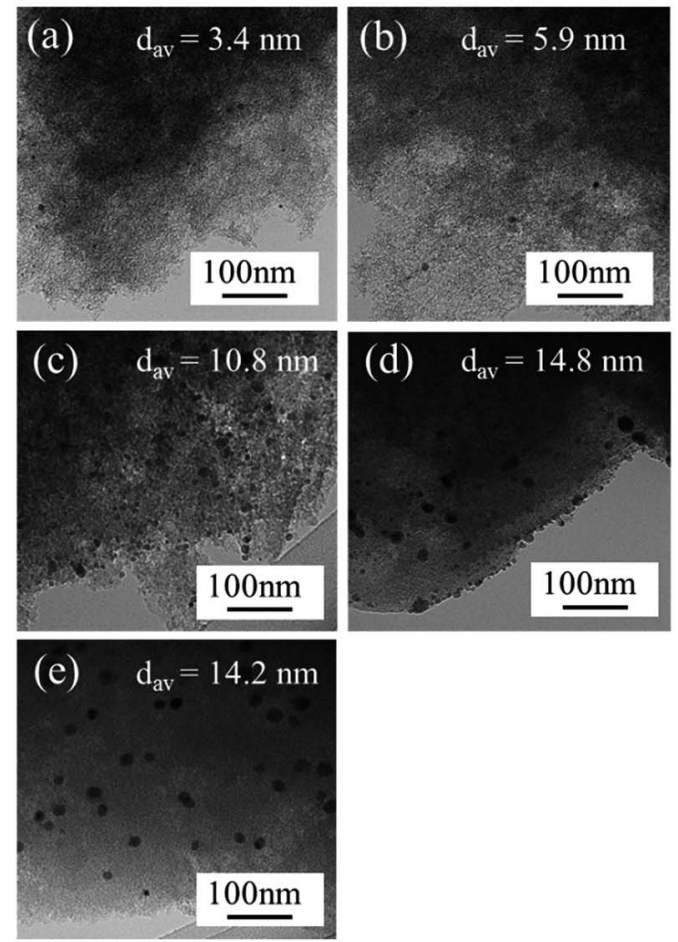

Fig. 2 TEM images of (A) Co/AC- $T$ ( $T=$ (a) 400, (b) 500, (c) 600, (d) 800 ) and (B) PdCo/AC-T-GR ( $T=$ (a) 400, (b) 500, (c) 600, (d) 800) and (e) PdCo/AC-600-imp.

AC-600 and Co/AC-800 samples contained NPs with a mean diameter of $13.0 \mathrm{~nm}$ and $18.8 \mathrm{~nm}$, respectively. These TEM images, together with the XRD results, indicate that NPs are produced above $500{ }^{\circ} \mathrm{C}$ and that the diameter of the NPs increases as the heat treatment temperature increases. TEM images of PdCo/AC-T-GR and PdCo/AC-600-imp subjected to hydrogen reduction at $200{ }^{\circ} \mathrm{C}$ for $1 \mathrm{~h}$ are shown in Fig. 2(B). $\mathrm{PdCo} / \mathrm{AC}-400-\mathrm{GR}$ contained clear NPs with a mean diameter of $3.4 \mathrm{~nm}$, in contrast to $\mathrm{Co} / \mathrm{AC}-400$, which suggests the formation of Pd or Pd-Co NPs by the GR method, followed by hydrogen reduction. PdCo/AC-500, -600 and -800 also contained distinct 
NPs with a mean diameter of $5.9 \mathrm{~nm}, 10.8 \mathrm{~nm}$ and $14.8 \mathrm{~nm}$, respectively. The size of the NPs in PdCo/AC-500-GR was similar to that of $\mathrm{Co} / \mathrm{AC}-500$, whereas the PdCo/AC-600-GR and -800-GR NPs were slightly smaller than those of their Co/AC- $T$ counterparts. This finding suggests that Co NPs are replaced by Pd species while maintaining the size of the original Co NPs throughout the GR process. Thus, the size of the NPs in PdCo/ AC- $T$-GR is primarily determined by the heat treatment temperature during $\mathrm{Co} / \mathrm{AC}-\mathrm{T}$ synthesis. PdCo/AC-600-imp as a reference sample provided NPs with a mean diameter of $14.2 \mathrm{~nm}$.

The crystallinity and composition of the NPs in PdCo/AC600-GR were investigated by scanning transmission electron microscopy (STEM). Fig. 3 shows a high-angle annular darkfield (HAADF) STEM image and EDX elemental mapping images of PdCo/AC-600-GR. Regularly aligned fringes of the Pd fcc structure are partly visible in the NPs in the HAADF-STEM image, but fringes are not discernible in most areas of the NPs, indicating their low degree of crystallization, consistent with the XRD results. The EDX elemental mapping images indicate that the NPs are mainly composed of Pd species. Co species are segregated in the vicinity of the NPs and are also highly dispersed over the surface of the carbon support.

X-ray absorption fine structure (XAFS) measurements were performed to investigate the more detailed local structure of the Pd species. Fig. 4 shows the Pd K-edge X-ray absorption nearedge structure (XANES) and Fourier transformed extended Xray absorption fine structure (FT-EXAFS) spectra of PdCo/ACT-GR, PdCo/AC-600-imp, and reference samples. The peak shapes in the XANES spectra of a series of PdCo/AC- $T$-GR are similar to those for Pd foil. The FT-EXAFS spectra of all PdCo/ AC-T-GR samples exhibit a strong peak at around $2.6 \AA$, attributed to Pd-Pd bonds, revealing the presence of Pd NPs. No clear
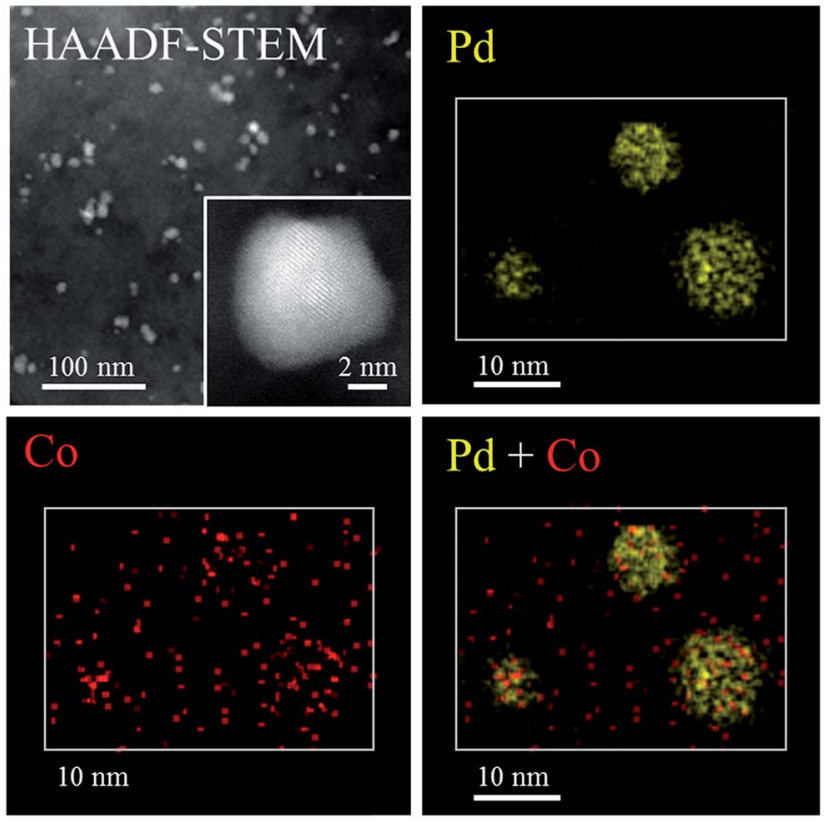

Fig. 3 HAADF-STEM image and EDX mapping of PdCo/AC-600-GR.
(A)

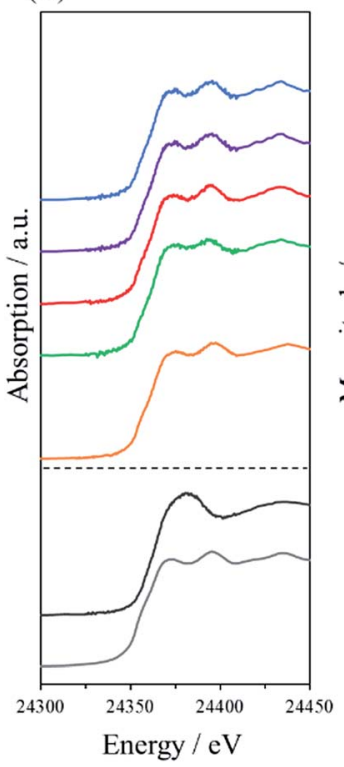

(B)

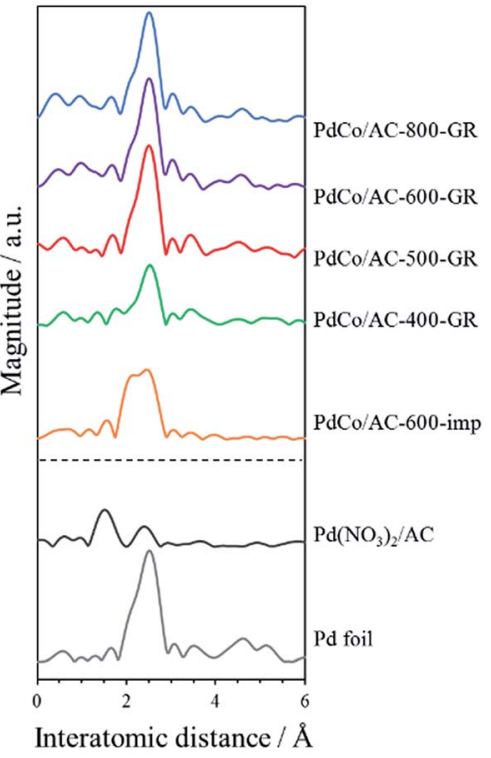

Fig. $4 \mathrm{Pd}$ K-edge (A) XANES spectra and (B) FT-EXAFS spectra of $\mathrm{PdCo} / \mathrm{AC}-\mathrm{T}-\mathrm{GR}, \mathrm{PdCo} / \mathrm{AC}-600$-imp and reference samples.

peak attributed to the Pd-Co bond (typically seen at $2.2 \AA)^{26}$ is observed in any PdCo/AC-T-GR sample. On the other hand, PdCo/AC-600-imp shows two strong peaks, at $2.2 \AA$ and $2.6 \AA$, which can be attributed to $\mathrm{Pd}-\mathrm{Co}$ and $\mathrm{Pd}-\mathrm{Pd}$ bonds, respectively. This supports the formation of a Pd-Co bimetallic alloy, consistent with the XRD result.

The above characterization results indicate that $\mathrm{Pd}^{2+}$ ions displace Co in Co/AC- $T$ during the GR process, followed by the formation of partially crystallized Pd NPs containing a low concentration of Co in all samples of PdCo/AC-T-GR. The size of the original Co NPs does not change significantly during the GR process. At the same time, highly dispersed displaced Co species are deposited on the surface of the carbon support. Previous use of the GR method for catalyst preparation indicated that the Pd species was replaced on the surface of the Co NPs with high dispersion, resulting mainly in the formation of Co@Pd core-shell catalysts. ${ }^{32-34}$ In contrast, in the present preparation, Co NPs are almost fully displaced by Pd species in all samples, and Pd-rich NPs are produced rather than Co@Pd core-shell NPs, probably due to the small size of the original Co NPs.

\section{Catalytic test}

The catalytic performance of PdCo/AC-T-GR was examined in the hydrogenation of phenylacetylene. This is a sequential reaction in which styrene, produced from phenylacetylene, is successively hydrogenated into ethylbenzene. Fig. 5 shows the catalytic performance of PdCo/AC-T-GR $(T=400,500,600$ and $800)$, evaluated by the conversion of phenylacetylene and the selectivity for styrene. As the heat treatment temperature during $\mathrm{Co} / \mathrm{AC}-T$ preparation increased from 400 to $600{ }^{\circ} \mathrm{C}$, the activity and selectivity increased, whereas PdCo/AC-800-GR exhibited lower activity and selectivity than PdCo/AC-600-GR. Activity and 


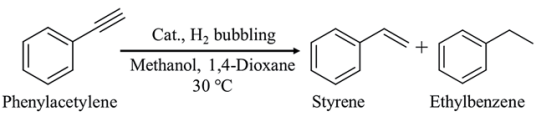

(A)

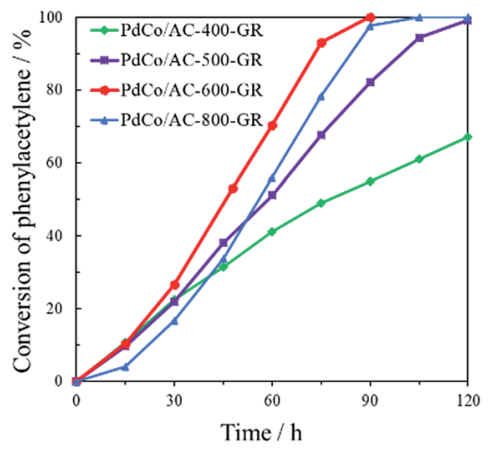

(B)

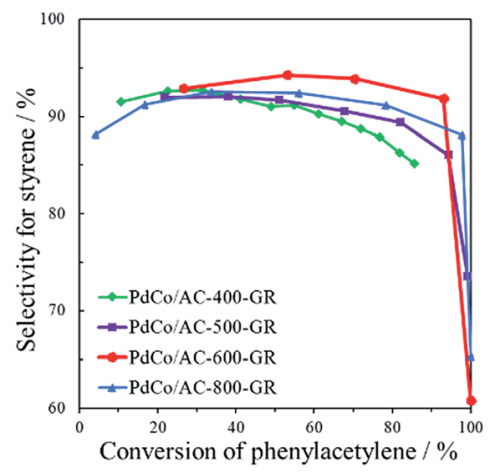

Fig. 5 (A) Reaction profiles and (B) conversion-selectivity relationship in the hydrogenation of phenylacetylene over PdCo/AC- $T$-GR $(T=$ $400,500,600,800)$.

selectivity were altered by the heat treatment temperature of PdCo/AC-T-GR, and PdCo/AC-600-GR showed the highest conversion and selectivity among the PdCo/AC-GR- $T$ samples: $92 \%$ selectivity and $93 \%$ conversion after 75 min reaction.

In order to examine the reason for the different catalytic performance in a series of PdCo/AC-T-GR, the electronic properties of the Pd species in the catalysts were analyzed by XPS measurements. The Pd 3d spectra of PdCo/AC-T-GR are shown in Fig. 6. PdCo/AC-400-GR showed a binding energy (BE) peak of Pd $3 \mathrm{~d}_{5 / 2}$ at $336.0 \mathrm{eV}$. As the heat treatment temperature increased, the $\mathrm{BE}$ of PdCo/AC-500-GR and PdCo/AC-600-GR decreased (335.4 and $335.1 \mathrm{eV}$, respectively), indicating that the Pd species in $\mathrm{PdCo} / \mathrm{AC}-600$ were in a more electron-rich state compared with the case for PdCo/AC-400. On the other hand, the $\mathrm{BE}$ for $\mathrm{PdCo} / \mathrm{AC}-800$ was $335.3 \mathrm{eV}$, which is higher than that of PdCo/AC-600. These electronic states of a series of $\mathrm{PdCo} / \mathrm{AC}-\mathrm{T}$-GR were probably influenced by the remaining Co species incorporated in NPs. Fig. 7 shows the relationship between the electronic state (represented by the BE peak of the Pd $3 \mathrm{~d}_{2 / 5}$ spectra of PdCo/AC-T-GR (Fig. 6)) and catalytic activity and selectivity. A clear correlation between the electronic properties and catalytic performance was observed, and catalysts with more electron-rich Pd exhibited higher catalytic activity and selectivity in the selective hydrogenation reaction. It

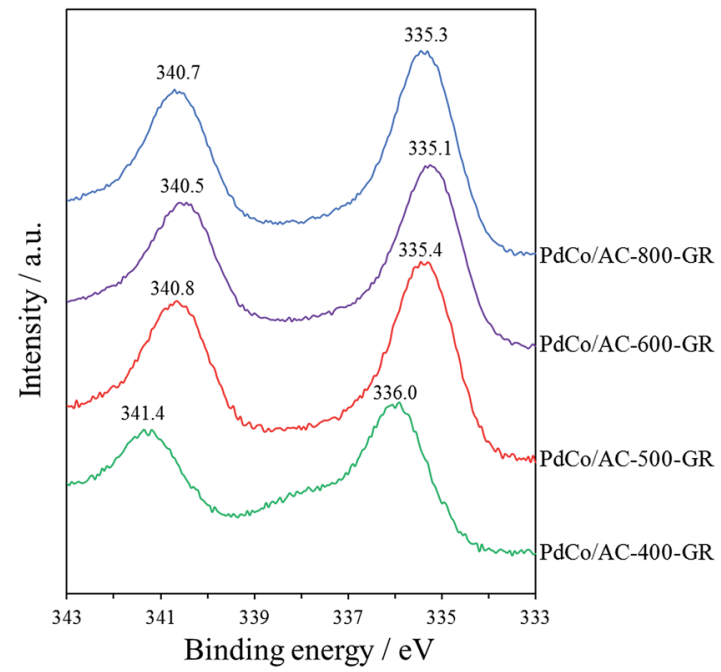

Fig. 6 Pd 3d XPS spectra of PdCo/AC-T-GR, PdCo/AC-600-imp.

is suggested that the Pd-Co ensembles with electron-rich Pd act as suitable active sites for selective hydrogenation of phenylacetylene. The electron richness of Pd would promote the charge transfer into the $\pi^{*}$ orbitals and destabilization of triple bond of phenylacetylene, which accelerate the hydrogenation of phenylacetylene to yield styrene. ${ }^{41}$ On the other hand, the interaction between electron-rich $\pi$-bond of styrene and the electron-rich Pd species is not as strong as that of the electrondeficient Pd species. Therefore, the electron-rich Pd species exhibit a lower adsorption energy toward styrene, which probably suppresses the sequential hydrogenation of styrene to yield ethylbenzene. ${ }^{31}$ This is the probable reason for the high activity and selectivity of PdCo/AC-600-GR possessing electron-rich Pd species.

Fig. 8 shows the catalytic performance of PdCo/AC-600-GR and the reference catalysts. Co/AC-600 exhibited no catalytic activity in this reaction, whereas Pd/AC showed $80 \%$ selectivity and $90 \%$ conversion after $105 \mathrm{~min}$ reaction, which is about $12 \%$ lower selectivity than that of PdCo/AC-600-GR. These results reveal that Pd is active site for the hydrogenation of phenylacetylene in PdCo/AC-600-GR, while selectivity is enhanced by

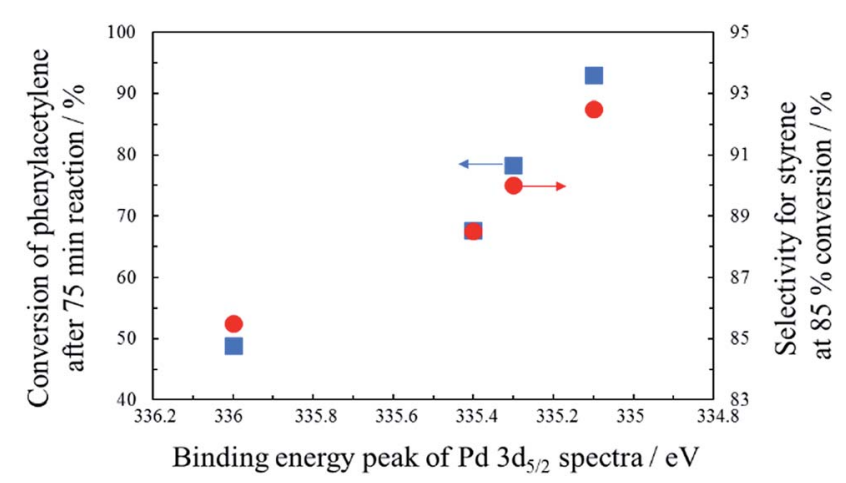

Fig. 7 The relationship between the electronic state of $\mathrm{Pd}$ and conversion of phenylacetylene/selectivity for styrene. 


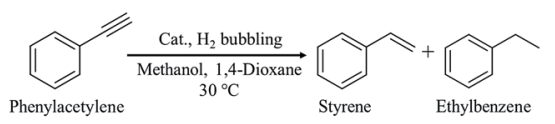

(A)

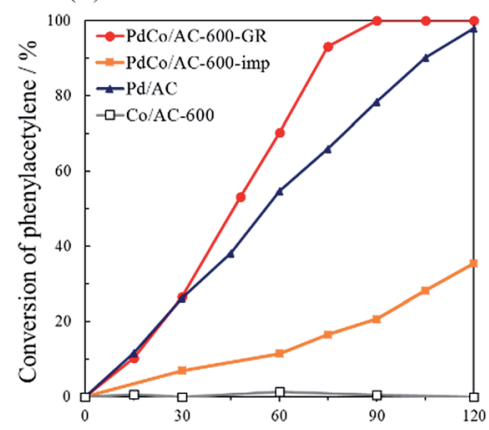

(B)

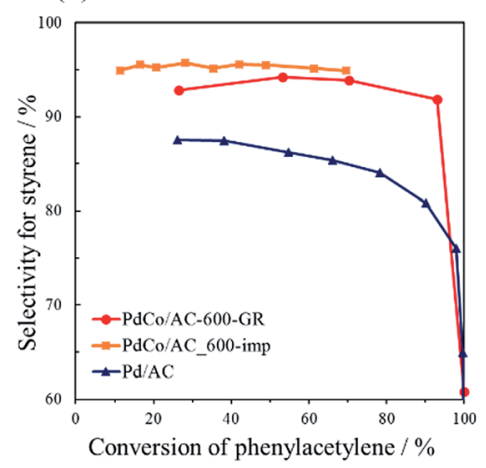

Fig. 8 (A) Reaction profiles and (B) conversion-selectivity relationship in the hydrogenation of phenylacetylene over PdCo/AC-600-GR, $\mathrm{PdCo} / \mathrm{AC}-600$-imp, Pd/AC and Co/AC-600.

the addition of Co species. PdCo/AC-600-imp showed nearly the same selectivity as PdCo/AC-600-GR, but lower activity than PdCo/AC-600-GR, probably due to the low concentration of surface Pd species.

\section{Conclusions}

We adopted the GR method for the synthesis of Pd-Co NP catalysts using Co NPs with various sizes and tested their catalytic performance for the selective hydrogenation of phenylacetylene. Carbon-supported Co species ranging from singlesite to NPs were prepared by simply varying heat treatment temperature, and subsequently replaced with $\mathrm{Pd}^{2+}$ ions via the GR process. In contrast to previously reported results, the formed NPs were mainly composed of Pd species, with Co species present at low concentration. The size of the NPs did not change significantly during the GR process, as judged from the TEM images. The electronic properties of the Pd species were dependent on the size of the Pd-Co NPs, as determined from the XPS results. We found a correlation between the electronic state of the Pd species and catalytic activity/selectivity in the selective hydrogenation of phenylacetylene: PdCo/AC-600-GR, possessing the most electron-rich Pd species, showed the best catalytic performance, and provided 92\% selectivity at 93\% conversion. Moreover, the selectivity of PdCo/AC-600-GR was higher than that of pure Pd catalyst and its activity was higher than that of a Pd-Co alloy catalyst.

\section{Acknowledgements}

The present work was supported by the Grants-in-Aid for Scientific Research (KAKENHI, No. 26220911 and 26620194) from the Japan Society for the Promotion of Science (JSPS).

\section{Notes and references}

1 N. Toshima and T. Yonezawa, New J. Chem., 1998, 22, 1179. 2 L. Peng, E. Ringe, R. P. Van Duyne and L. D. Marks, Phys. Chem. Chem. Phys., 2015, 17, 27940.

3 W. Xu, X. Liu, J. Ren, P. Zhang, Y. Wang, Y. Guo, Y. Guo and G. Lu, Catal. Commun., 2010, 11, 721.

4 W. Yu, M. D. Porosoff and J. G. Chen, Chem. Rev., 2012, 112, 5780 .

5 K. Fuku, T. Sakano, T. Kamegawa, K. Mori and H. Yamashita, J. Mater. Chem., 2012, 22, 16243.

6 K. Mori, K. Miyawaki and H. Yamashita, ACS Catal., 2016, 6, 3128.

7 F. Gao and D. W. Goodman, Chem. Soc. Rev., 2012, 41, 8009. 8 B. Coq and F. Figueras, J. Mol. Catal. A: Chem., 2001, 173, 117. 9 C. Huang, H. Zhang, Y. Zhao, S. Chen and Z. Liu, J. Colloid Interface Sci., 2012, 386, 60.

10 X. Liu, D. Wang and Y. Li, Nano Today, 2012, 7, 448.

11 M. Oezaslan, M. Heggen and P. Strasser, J. Am. Chem. Soc., 2012, 134, 514.

12 Z. Wang, L. Yang, R. Zhang, L. Li, Z. Cheng and Z. Zhou, Catal. Today, 2016, 264, 37.

13 Y. Xu, L. Chen, X. Wang, W. Yao and Q. Zhang, Nanoscale, 2015, 7, 10559.

14 A. Villa, D. Wang, D. S. Su and L. Prati, Catal. Sci. Technol., 2015, 5, 55.

15 Z. M. Adriana, M. Martyna, D. Magdalena and G. Ewelina, Adv. Colloid Interface Sci., 2016, 229, 80.

16 A. Dehghan Banadaki and A. Kajbafvala, J. Nanomater., 2014, 2014, 985948.

17 W. G. Menezes, L. Altmann, V. Zielasek, K. Thiel and M. Bäumer, J. Catal., 2013, 300, 125.

18 L. Zhang, Z. Xie and J. Gong, Chem. Soc. Rev., 2016, 45, 3916. 19 S. M. Alia, Y. Yan and B. Pivovar, Catal. Sci. Technol., 2014, 4, 3589.

20 X. Xia, Y. Wang, A. Ruditskiy and Y. Xia, Adv. Mater., 2013, 25, 6313.

21 L. Jiang, A. Hsu, D. Chu and R. Chen, Electrochim. Acta, 2010, 55, 4506.

22 C. L. Lee and C. M. Tseng, J. Phys. Chem. C, 2008, 112, 13342.

23 C. L. Lee, C. M. Tseng, R. B. Wu, C. C. Wu and S. C. Syu, Electrochim. Acta, 2009, 54, 5544.

24 S. M. Alia, K. Duong, T. Liu, K. Jensen and Y. Yan, ChemSusChem, 2014, 7, 1739.

25 S. M. Alia, B. S. Pivovar and Y. Yan, J. Am. Chem. Soc., 2013, 135, 13473.

26 M. Shao, K. Sasaki, N. S. Marinkovic, L. Zhang and R. R. Adzic, Electrochem. Commun., 2007, 9, 2848. 
27 S. M. Alia, K. O. Jensen, B. S. Pivovar and Y. Yan, ACS Catal., 2012, 2, 858.

28 H. Li, H. Lin, Y. Hu, H. Li, P. Li and X. Zhou, J. Mater. Chem., 2011, 21, 18447.

29 M. B. Boucher, B. Zugic, G. Cladaras, J. Kammert, M. D. Marcinkowski, T. J. Lawton, E. C. H. Sykes and M. Flytzani-Stephanopoulos, Phys. Chem. Chem. Phys., 2013, 15, 12187.

30 S. Okada, T. Kamegawa, K. Mori and H. Yamashita, Catal. Today, 2012, 185, 109.

31 Y. Zhang, W. Diao, J. R. Monnier and C. T. Williams, Catal. Sci. Technol., 2015, 5, 4123.

32 K. Shen, L. Chen, J. Long, W. Zhong and Y. Li, ACS Catal., 2015, 5, 5264.

33 J. Ma, L. Xu, L. Xu, H. Wang, S. Xu, H. Li, S. Xie and H. Li, ACS Catal., 2013, 3, 985.
34 Y. Mahara, J. Ohyama, T. Tojo, K. Murata, H. Ishikawa and A. Satsuma, Catal. Sci. Technol., 2016, 6, 4773.

35 H. Zhang, M. Jin, H. Liu, J. Wang, M. J. Kim, D. Yang, Z. Xie, J. Liu and Y. Xia, ACS Nano, 2011, 5, 8212.

36 T. Fukuda and T. Katsuki, Tetrahedron, 1997, 53, 7201.

37 K. Nakatsuka, T. Yoshii, Y. Kuwahara, K. Mori and H. Yamashita, Phys. Chem. Chem. Phys., 2017, 19, 4967.

38 S. Navaladian, B. Viswanathan, T. K. Varadarajan and R. P. Viswanath, Nanoscale Res. Lett., 2009, 4, 181.

39 L. Zhang, K. Lee and J. Zhang, Electrochim. Acta, 2007, 52, 3088.

40 R. N. Singh and C. S. Sharma, Eng. Tech. Appl. Sci. Res., 2012, 2, 295.

41 G. X. Pei, X. Y. Liu, A. Wang, A. F. Lee, M. A. Isaacs, L. Li, X. Pan, X. Yang, X. Wang, Z. Tai, K. Wilson and T. Zhang, ACS Catal., 2015, 5, 3717. 